\title{
Pancytopenia in a child with cystic fibrosis and severe copper deficiency: Insight from bone marrow evaluation
}

\author{
Maggie Seblani ${ }^{1}$, Susanna McColley ${ }^{2}$, Shunyou Gong ${ }^{1}$, Lee Bass $^{2}$, and Sherif Badawy ${ }^{2}$ \\ ${ }^{1}$ Ann and Robert H Lurie Children's Hospital of Chicago \\ ${ }^{2}$ Northwestern University Feinberg School of Medicine
}

June 23, 2021

Title Page

Title : Pancytopenia in a child with cystic fibrosis and severe copper deficiency: Insight from bone marrow evaluation

Authors : Maggie D. Seblani ${ }^{1,2}$; Susanna A. McColley ${ }^{2,3,4}$; Shunyou Gong ${ }^{5}$; Lee M. Bass ${ }^{2,6}$; Sherif M. Badawy $^{1,2}$

Affiliations : ${ }^{1}$ Division of Hematology, Oncology and Stem Cell Transplant, Ann \& Robert H. Lurie Children's Hospital of Chicago, Chicago, IL, USA; ${ }^{2}$ Department of Pediatrics, Northwestern University Feinberg School of Medicine, Chicago, IL, USA; ${ }^{3}$ Division of Pulmonary and Sleep Medicine, Ann \& Robert H. Lurie Children's Hospital of Chicago, Chicago, IL, USA; ${ }^{4}$ Stanley Manne Children's Research Institute, Ann \& Robert H. Lurie Children's Hospital of Chicago, Chicago, Illinois, USA; ${ }^{5}$ Division of Pediatric Pathology, Ann \& Robert H. Lurie Children's Hospital of Chicago, Chicago, IL; ${ }^{6}$ Division of Gastroenterology, Hepatology and Nutrition, Ann \& Robert H. Lurie Children's Hospital of Chicago, Chicago, IL.

Correspondence: Sherif M. Badawy, MD, MS, MBBCh, 225 E. Chicago Ave., Box \#30, Chicago, IL, 60611, office: 312-227-4836, fax: 312-227-9376, e-mail: sbadawy@luriechildrens.org

Word count: 498

Number of Tables, Figures, and Supplemental files : 1

Running title: Bone marrow findings with severe copper deficiency

Keywords: pancytopenia, anemia, leucopenia, neutropenia, thrombocytopenia, copper deficiency, ring sideroblasts, copper supplement, cystic fibrosis, malabsorption

Conflict of Interest: Author has no conflicts of interest to disclose.

\section{To the Editor}

We present a case of a nine-year-old male with cystic fibrosis, who underwent hepato-portoenterostomy for biliary dysplasia and jejunal resection for distal intestinal obstruction syndrome, requiring chronic parenteral nutritional support. He required multiple transfusions for episodes of severe anemia and thrombocytopenia and was referred to hematology for further evaluation. At initial visit he had macrocytic anemia (hemoglobin 7.3, reference range 11.5 - $13.5 \mathrm{~g} / \mathrm{dL}$; and mean corpuscular volume [MCV] 96.1, (reference range 75-87 fl), mild leukopenia (white blood cell count $[\mathrm{WBC}] 4.02$, reference range $6.0-17.0$ thou/uL) and severe neutropenia (absolute neutrophil count [ANC] 0.442, reference range $0.72-7.525$ thou/uL). While no thrombocytopenia at visit, platelet counts previously ranged from 43 to 148 (reference range 150 - 450 thou/uL). 
On physical exam, significant findings were short stature at less than fifteenth percentile for height, pallor, mild hepatosplenomegaly and lower extremity weakness

Evaluation for his pancytopenia included Coombs and anti-neutrophil antibody as destructive etiologies, which were both negative. Given concern for nutritional deficiency, folate, vitamin B12, methylmalonic acid, and vitamin $\mathrm{E}$ levels were obtained in addition to ferritin, serum iron, saturation \%, total iron binding capacity and soluble transferrin receptor, which were all unremarkable. There were no significant morphologic abnormalities noted on peripheral blood smear. With unrevealing laboratory evaluation and in the context of his mild organomegaly, anemia and thrombocytopenia were attributed to hypersplenism. Due to persistent pancytopenia, notably WBC 1.31 thou/uL, hemoglobin $5.8 \mathrm{~g} / \mathrm{dL}$, MCV $94.9 \mathrm{fl}$, platelet count 116 thou/uL and ANC 0.168 thou/uL, a bone marrow aspirate and biopsy evaluation was pursued. While there was progressive myelopoiesis and no dysplastic features, his marrow showed findings of vacuolated granules and ring sideroblasts, which were suggestive of possible copper deficiency (Figure 1 ). His serum copper level was very low at less than $5 \mathrm{mcg} / \mathrm{dL}$ (reference range $117-181 \mathrm{mcg} / \mathrm{dL}$ ) and serum ceruloplasmin level was less than $3 \mathrm{mg} / \mathrm{dL}$ (reference range 25-52 mg/dL, for males 7-9 years). The patient was diagnosed with severe copper deficiency and replacement therapy with daily enteral copper gluconate $2 \mathrm{mg}$ was initiated.

Poor absorption from underlying cystic fibrosis complicated by intestinal resection were primary contributors to the patient's copper deficiency. Additionally, prolonged parenteral nutrition and prior zinc supplementation may have compounded his deficiency, related to zinc's antagonistic relationship to copper metabolism and absorption. ${ }^{1}$ Two months following initiation of enteral supplementation, his serum copper and ceruloplasmin levels normalized. With ongoing supplementation, he had remarkable and sustained improvement of his blood counts including hemoglobin ranging from 11.7 to $14.8 \mathrm{~g} / \mathrm{dL}$, normalized MCV, WBC of 4.01 to 8.09 thou/uL, improvement in ANC ranging from 1.620 to 5.20 thou/uL as well as maintenance of a platelet count between 77 and 181 thou/uL.

In conclusion, our patient highlights the importance of assessment for copper deficiency in a patient presenting with pancytopenia, especially those with risk factors such as poor intestinal absorption or chronic parenteral nutrition. Our patient had a remarkable count recovery quickly following initiation of copper supplementation with no further transfusion needs.

\section{Figure Legend:}

Figure 1: Bone marrow aspirate findings consistent with copper deficiency. (A) Frequent myeloid and erythroid precursor cells with cytoplasmic vacuoles (indicated by arrows) as well as a few nucleated red blood cells with nuclear irregularity in the background (Wright-Giemsa stain, original magnification x 1,000); (B)Occasional ring sideroblasts (indicated by arrows), suggestive of mitochondrial iron deposits (Prussian blue stain, original magnification x 1,000).

\section{References:}

Wapnir RA. Copper absorption and bioavailability. Am J Clin Nutr. 1998;67(5 Suppl):1054s-60s 


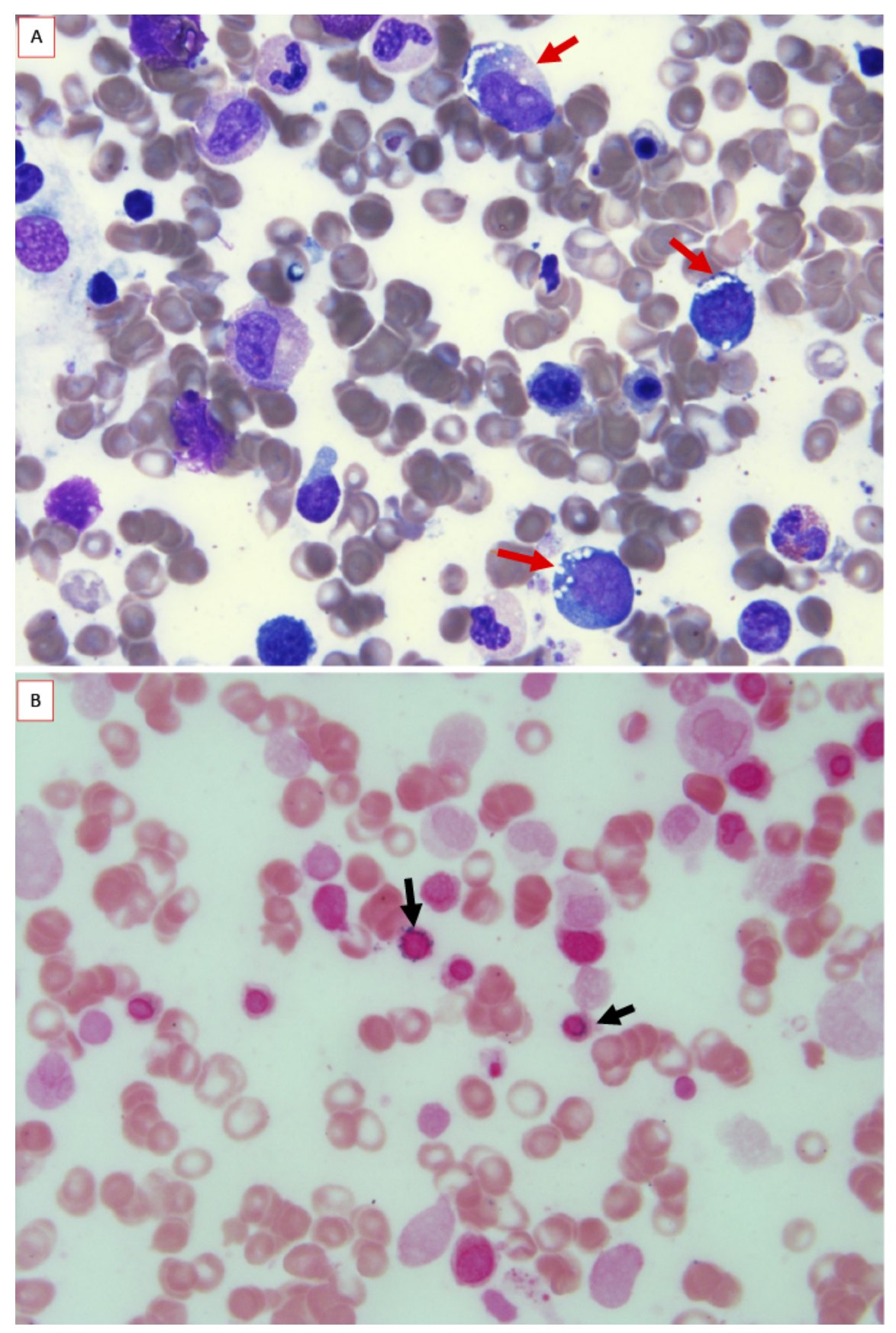

